\title{
Inelastic torsional seismic response of nominally symmetric reinforced concrete frame structures: Shaking table tests
}

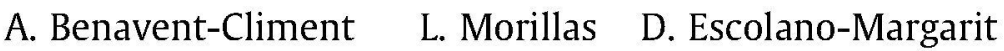

\begin{abstract}
A B S T R A C T
This paper discusses the torsional response of a scaled reinforced concrete frame structure subjected to several uniaxial shaking table tests. The tested structure is nominally symmetric in the direction of shaking and exhibits torsion attributable to non-uniform yielding of structural components and uncertainties in the building process. Asymmetric behavior is analyzed in terms of displacement, strain in reinforcing bars, energy dissipated at plastic hinges, and damage at section and frame levels. The results show that for low levels of seismic hazard, for which the structure is expected to perform basically within the elastic range, the accidental eccentricity is not a concern for the health of the structure, but it significantly increases the lateral displacement demand in the frames (about 30\%) and this might cause significant damage to non-structural components. For high levels of seismic hazard the effects of accidental torsion become less important. These results underline the need to consider accidental eccentricity in evaluating the performance of a structure for very frequent or frequent earthquakes, and suggest that consideration of torsion may be neglected for performance levels associated with rare or very rare earthquakes.
\end{abstract}

\section{Introduction}

In the calculation of seismic forces in structural members, it is common to shift the centers of masses just a percentage of the dimension of the building perpendicular to the ground motion and away from the center of lateral stiffness. This shift is known as accidental design eccentricity (ADE). Its value is typically $5 \%$ and it is used to indirectly account for a number of phenomena, such as effects of the rotational components of ground motion, non-uniform yielding of structural components, stiffness degradation of parts of the lateral resisting system, or uncertainties in mass distribution and structural properties. The value and the concept of ADE have been a matter of debate since this static approach was introduced in seismic codes. Some authors $[1,2]$ consider that the application of ADE deserves reexamination because the mere shifting of the center of mass hardly represents the complexity of the mentioned phenomena, and because it leads to insignificant changes in the building design. Some other reasons why the concept of $A D E$ is in dispute are that: (i) it is known that the increase in building response due to accidental eccentricity does not depend on the building dimension [3]; (ii) mass eccentricities of the order usually prescribed by codes are not important when the building response is strongly inelastic [2]; (iii) ductility demands in buildings designed with and without considering accidental eccentricity show marginal or negligible variations [2]; (iv) results may not be applicable to cases where yielding occurs $[2,4]$; or (v) design eccentricity increases the translational resistance but provides no torsional resistance [5]. Works by Marušić and Fajfar [6] or Kosmopoulos and Fardis [7] suggest that effects of torsion be better described as the amplification of the displacement demand due to torsion, or in terms of chord rotations. These approaches are especially interesting in the framework of Performance Based Seismic Design, as it focuses on the assessment of the response rather than on the resistance of the structure. Nonetheless, the simple and conceptually attractive application of ADE is found in code provisions worldwide [8]. Meanwhile, the assessment of an adequate value for design eccentricity to account for accidental torsion has been a matter of study, and over recent decades several issues have been addressed separately in literature.

In his early studies, Newmark [4] first called into question whether an ADE of $5 \%$ is enough to account for the effects of torsional ground motions in (symmetrical) buildings, and recommended great conservatism in the design of corner columns and end shear walls. New data proved that some of Newmark's assumptions on torsional ground motion led him to excessive results, but whether a $5 \%$ of $\mathrm{ADE}$ is conservative or not is still 
controversial $[1,9,10]$. Although the issue of torsional excitations still awaits credible records to reach a consensus regarding their effects on the torsional response of the structure, several findings have been put forth. It has been suggested that accidental torsion effects: (i) be generally larger for symmetric plans than for asymmetric plans $[2,4,11,12]$; (ii) be governed by the ratio of translational to lateral periods $\Omega[4,6,10,11-15]$; and (iii) be sensitive to dynamic characteristics, i.e. to the distinction between torsionally flexible and stiff systems $[2,6,10]$.

The uncertainty related to structural properties has been addressed by parametric studies changing the stiffness, strength, and position of the center of mass in numerical models. Uncertainties in stiffness may imply that a nominally symmetric system behaves as asymmetric to some unknown degree [13]. Escobar [16] singled out that it is not possible to judge torsional effects without considering structural performance, because the peak rotational and translational response do not occur at the same time. The effects of non-uniform yielding of structural components in accidental torsion have also been discussed in the literature. Authors in references $[1,4,17]$ share the opinion that: (i) accidental torsion may be significant for buildings that undergo yielding or local failures; (ii) yielding in torsion may be more serious than yielding in linear displacement; and (iii) the problem is considerably more complicated as a consequence of simplifications introduced in the static procedure. Analyses by Fajfar et al. [18] support that torsional effects decrease with an increase of plastic deformations, and that this is manifested mainly in smaller amplifications of displacement due to torsion on the flexible side of the structure. Extensive parametric studies on the response of symmetric and asymmetric buildings under biaxial excitation conducted by Marušić and Farfar [6] show that there is a small to moderate influence of ground motion intensity on the torsional amplification ratio, with values of about 1.3 for torsionally stiff structures.

Most knowledge surrounding ADE, as it is used in modern codes, comes from numerical studies either with elastic models or from simplified one-story three-degrees-of-freedom (DOF) inelastic models [18-22,23-26]. Experimental studies on the response of torsionally balanced or unbalanced reinforced concrete (RC) structures are limited, but those conducted with the wellknown SPEAR building are noteworthy $[27,28]$. The SPEAR building is likely to become the most-analyzed structure in earthquake engineering [27]. It was a full-scale three-storey plan-wise irregular RC frame structure representative of older constructions in southern European Countries, without specific provisions for earthquake resistance. It was subjected to a round of bi-directional pseudo-dynamic tests at the ELSA Laboratory of the Joint Research Centre. The results of the tests highlighted the strong effects of torsional irregularity on the column drifts, even for a limited level of plan eccentricity and relatively low levels of excitation. From the comparison between the outcomes of experimental results and the existing assessment procedures important drawbacks of the current codified approaches for the assessment of torsionally unbalanced multi-storey buildings were highlighted. This paper follows the experimental approach and discusses the results of several uniaxial shaking table tests conducted on a scaled $\mathrm{RC}$ frame structure up to collapse. The tested structure is nominally symmetric in the direction of shaking and it has no non-structural components. However, a torsional response was clearly observed during the tests, probably due to uncertainties in the building process and to non-uniform yielding of structural components. These uncertainties caused the structure to be stiffer on one side of the center of stiffness than on the other side. In this paper, key parameters involved in the torsional response are extracted from the empirical data and discussed. The results shed light on the effects of plastification of the structure on the severity of the torsional response. It is worth noting the differences between the tests conducted with the SPEAR building mentioned above, and those presented in this study. First, the former are pseudo-dynamic tests while the later are dynamic shaking table tests. Second, the former were conducted on a test structure irregular in plan which main source of torsional motion was the so-called "natural torsion", while the later was symmetric in the direction of shaking and the torsional response was governed by the "accidental eccentricity" attributable (mainly) to non-uniform yielding of structural components. Third, the SPEAR building represented a structure without specific provisions for earthquake resistance, while the one tested in this study represented a building designed for seismic loads.

\section{Experimental program}

\subsection{Test structure}

A three-story and three-bay RC moment-resisting frame was designed as prototype structure for this study and it is shown in Fig. 1. The prototype represents existing buildings in the Mediterranean area. It was designed applying limit state design methods, for the combined action of gravity and seismic loads. The dead

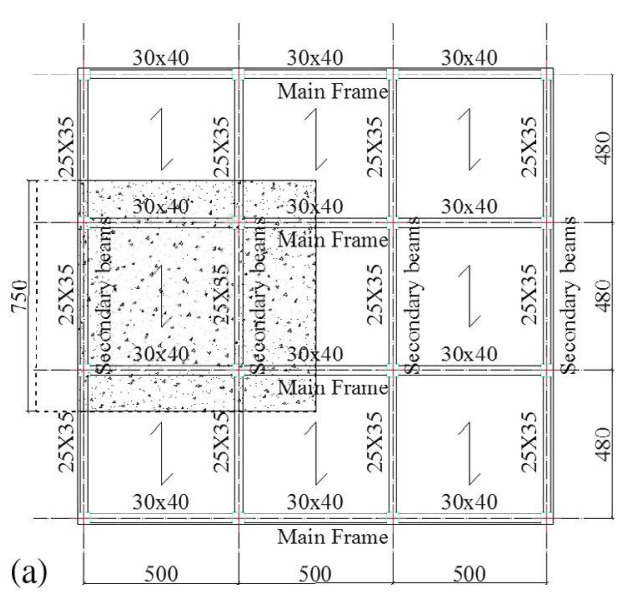

Partial structural model

Columns: $40 \times 40$

(b)

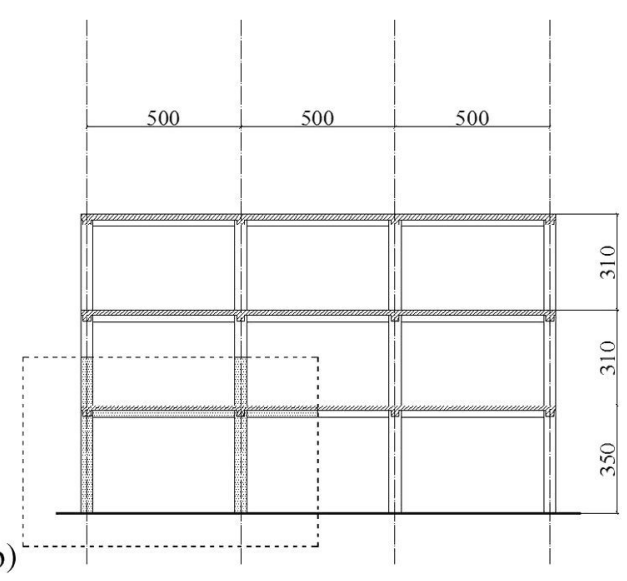

Fig. 1. Prototype buildings: (a) plan and (b) elevation. 


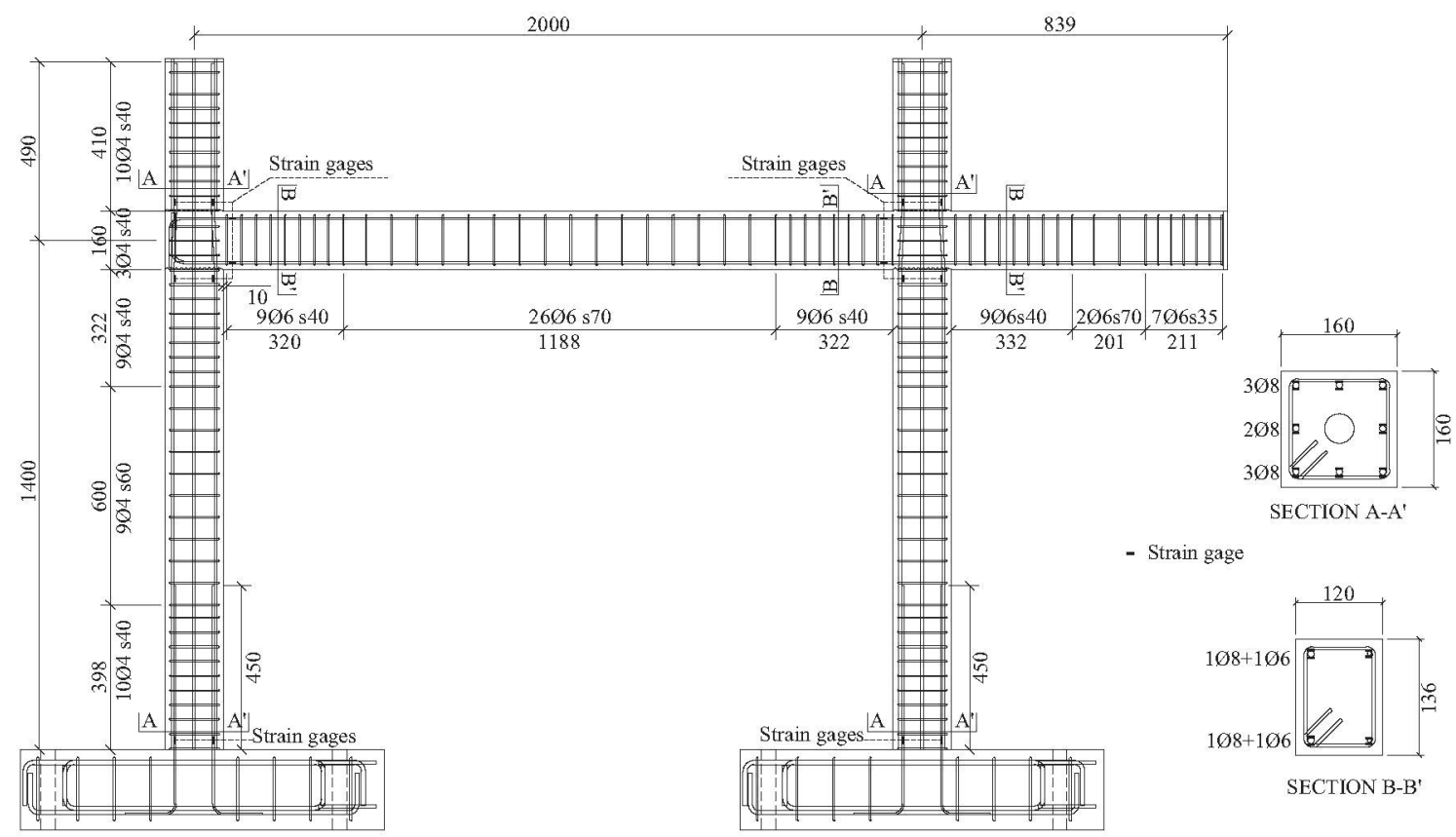

Fig. 2. Test specimen: elevation of a typical frame (dimensions in mm).

loads considered were $3.22 \mathrm{kN} / \mathrm{m}^{2}$ and $2.95 \mathrm{kN} / \mathrm{m}^{2}$ for floors and roof, respectively; and the live loads $2 \mathrm{kN} / \mathrm{m}^{2}$ and $1 \mathrm{kN} / \mathrm{m}^{2}$. The seismic design of the prototype followed the provisions of the current Spanish Seismic Code NCSE-02 [29]. Similarly to Eurocode-8 [30], the Spanish Seismic Code establishes that the calculated center of mass at each floor shall be considered as being displaced from its nominal location in each direction by an accidental eccentricity equal to 0.05 times the floor-dimension perpendicular to the direction of the seismic action. It was assumed that the prototype building is located on soft soil in the city of Granada (Spain). The design ground acceleration corresponding to this site and to this type of soil is $0.31 \mathrm{~g}$. This corresponds to the peak ground acceleration of the design earthquake having a return period $P_{r}=500$ years. The behavior factor considered in the seismic design was $q=3$, which corresponds to a structure that forms a "strong column-weak beam" mechanism under lateral loading.

From the prototype, a partial structural model was separated by cutting through points located at mid span of beams and at mid height of columns. Under the combination of seismic action with gravity loads prescribed by Eurocode 8 [30], the bending moments at these points are approximately zero. More precisely, in the case of the beams, the point of zero bending moment oscillates between 0.37 and 0.62 times the span of the beam. The partial structural model has the height of one story and a half, and the width of one bay and a half in the direction of the main beams. The test specimen was defined from the partial structural model by applying scale factors of $\lambda_{L}=2 / 5$ for length, $\lambda_{a}=1$ for acceleration and $\lambda_{\sigma}=1$ for stress. The test structure consists of two identical frames as shown in Fig. 2. The two frames are connected by a RC slab consisting of joists supporting a thin concrete plate reinforced with steel mesh, and perpendicular (secondary) beams, as seen in Fig. 3. A more detailed description of the test structure can be found in Benavent-Climent et al. [31].

\subsection{Test set-up}

The test structure was placed on a uniaxial MTS $3 \times 3 \mathrm{~m}^{2}$ shaking table at the University of Granada (Spain) as shown in Figs. 4 and 5 , so that the direction of shaking coincided with that of the

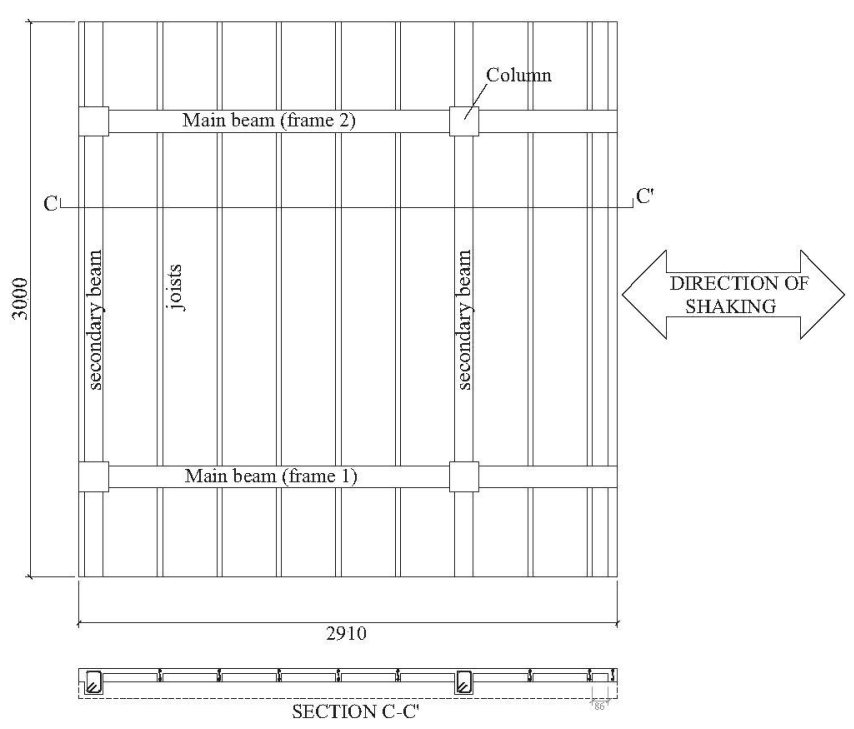

Fig. 3. Test specimen: plan (dimensions in $\mathrm{mm}$ ).

main beams, and with the axis of symmetry of the test structure. This was ensured through a significant control in the building and set-up process of the test structure. During the building process, the foundations were fixed to the floor of the Laboratory and the forms used for casting the concrete were fixed to the walls of the Laboratory. The foundations were linked by steel tubes to avoid relative displacements among them. Two openings on each foundation were used to fix the test structure to the shaking table by means of threaded rods and nuts. The position of these openings coincided with that of the threaded holes of the shaking table. A torque wrench was used to tighten the connecting nuts over the threaded rods. The applied torque was made large enough to prevent any relative displacement between the foundations and the shaking table during the dynamic tests. To represent the gravity loads acting on the floors and to satisfy scaling principles, steel blocks were attached at the top of the RC slab and at the top of half 


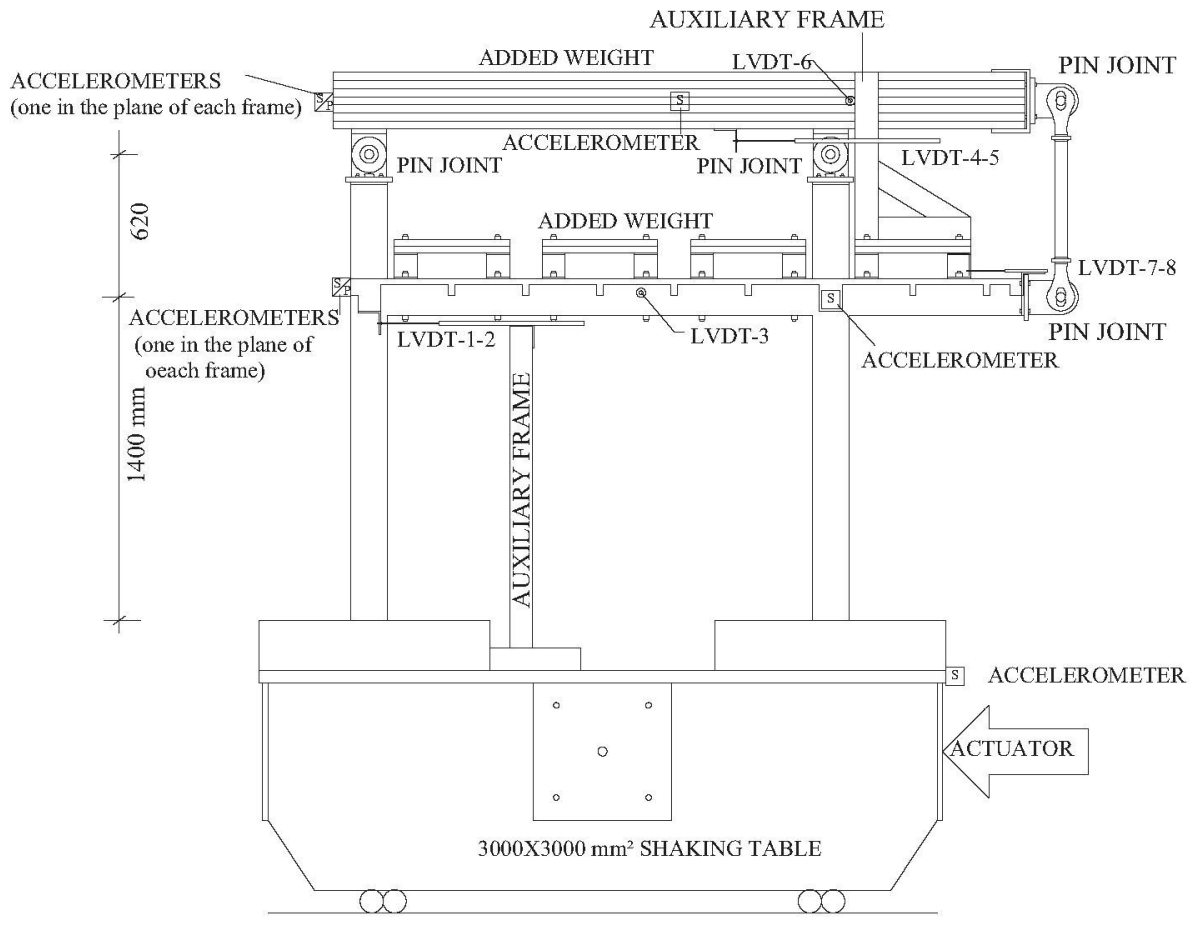

Fig. 4. Test set-up.

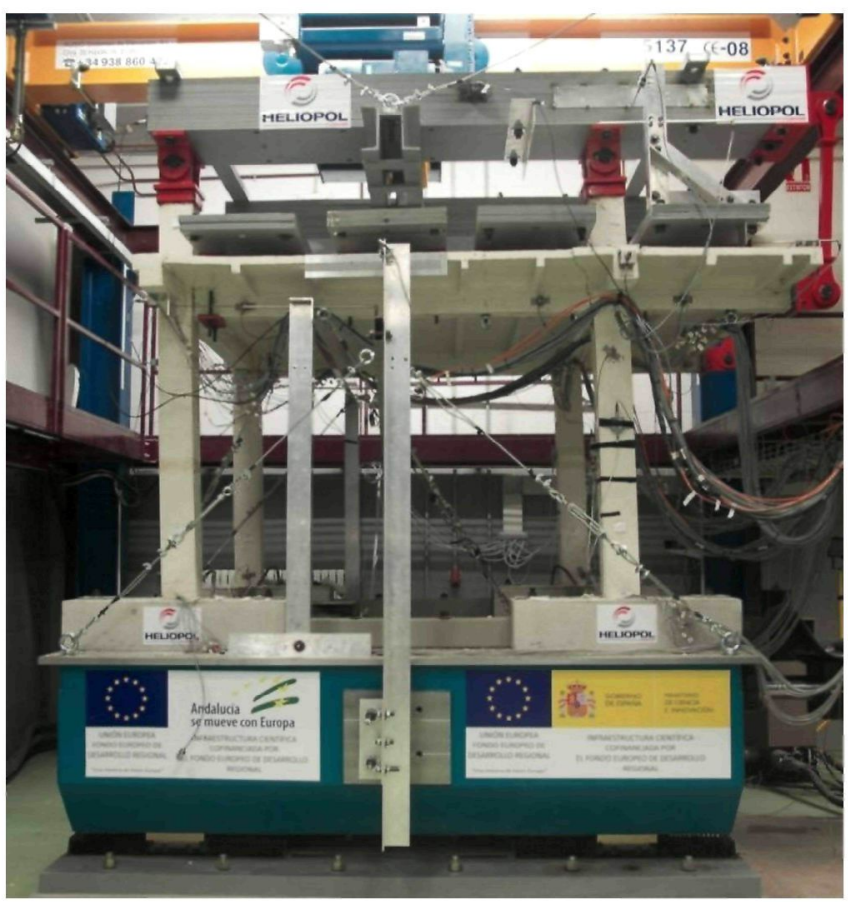

Fig. 5. Overall view of the tests.

columns of the second story, as shown in Fig. 4 (added weight). The boundary conditions (i.e. zero bending moment) of the test structure when the overall prototype building is subjected to lateral forces were reproduced by using pin joint connections at the top of the half-columns in the second story, and at the ends of the half-beams of the first floor. The vertical movements of the ends of the half-beams of the first floor were prevented by means of pin-ended steel bars -connecting the end of the beams with the steel plates (added weight) located atop the specimen - the steel plates have very large flexural stiffness in comparison with that of the RC slab. The total mass of the test specimen (including the additional masses) was $12,450 \mathrm{~kg}$. For idealizing the test structure with a lumped mass model explained later in Section 3.1, the mass of the foundations and the mass of half height of the columns of the first story is not considered, and this reduces the total mass to $10,070 \mathrm{~kg}$. It is worth noting that the purpose of the experiments was to investigate the behavior of the test specimen under earthquake-type dynamic loading, not to reproduce the particular response that the partial structural model would experience inside the overall frame under a particular ground motion, which is influenced by dynamic interactions with the upper part of the structure. That is, the tested partial structural model does not attempt to describe the response of the multi-story structure used as prototype. The specimen was instrumented with 192 strain gages, 10 uniaxial accelerometers and 9 displacement transducers. The measurements of the displacement transducers provided full information on the in-plane translations and rotations about a vertical axis, on the slab and on the steel blocks attached at the top of half columns of the second story. Similarly, the measurements of the accelerometers provided information on the in-plane absolute linear accelerations and angular accelerations about a vertical axis experienced by the slab and by the steel blocks attached at the top of half columns of the second story.

\subsection{Seismic simulations}

The test structure was subjected to dynamic tests that consisted of four seismic simulations referred to as $C 50, C 100, C 200$ and $C 300$ herein. In each seismic simulation the shaking table reproduced the ground motion recorded at Calitri during the Campano-Lucano (1980) earthquake. To fulfill the similitude laws, the original record was scaled in time by $\lambda_{\tau}=\sqrt{\lambda_{L} / \lambda_{a}}=0.63$. Fig. 6 shows the time history scaled in time by $\lambda_{\mathrm{t}}$ (Fig. $6 \mathrm{a}$ ) and the corresponding elastic response spectrum (Fig. 6b) of the applied dynamic loading. The elastic response spectrum has been normalized by the peak ground acceleration, $P G A$. To represent different seismic hazard levels 
(a)

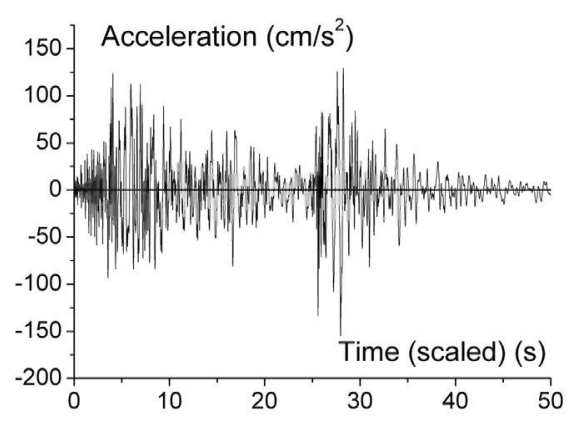

(b)

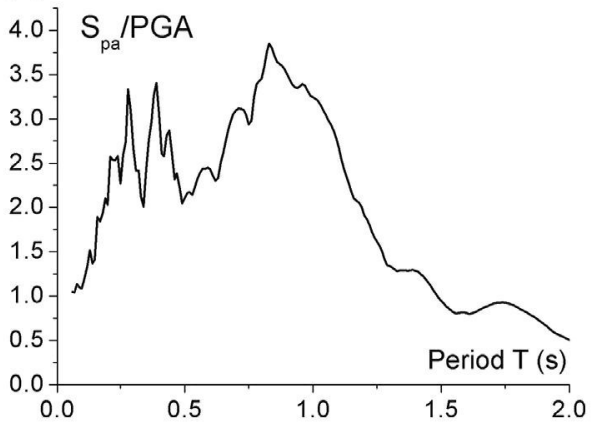

Fig. 6. Applied dynamic loading: (a) accelerogram and (b) elastic response spectra.

(SHL) the original ground motion was scaled in amplitude to $50 \%$, $100 \%, 200 \%$ and $300 \%$, in the seismic simulations C50, C100, C200 and $\mathrm{C} 300$, respectively. Their corresponding PGAs were $0.08 \mathrm{~g}$, $0.16 \mathrm{~g}, 0.31 \mathrm{~g}$ and $0.47 \mathrm{~g}$. Each $P G A$ represents a different SHL at the site (Granada) that will be referred to hereafter as SHL-1, SHL-2, SHL-3 and SHL-4. SHL-1 corresponds to a "very frequent" earthquake with $P_{r}=17$ years; SHL-2 a "frequent" earthquake with $P_{r}=97$ years; SHL-3 a "rare" earthquake with $P_{r}=500$ years; and SHL-4 a "very rare" or the "maximum considered" earthquake with $P_{r}=1435$ years. Free vibration tests were performed before and after each simulation.

\section{Test results and discussion}

\subsection{Idealization of the test structure}

For convenience in the forthcoming discussions, beams and columns of each of the two frames that constitute the specimen are idealized with macro models consisting of a linear elastic member connecting two plastic hinges at the ends that concentrate the inelastic flexural deformations, as shown in Fig. 7. Each plastic hinge is labeled with an identification number $k$. For the purpose of analyzing the dynamic response, the floor diaphragm with the added weight (referred to as "first level" herein), and the added weight put on the top of the columns (referred to as "second level" herein) are assumed to behave as two rigid blocks, which masses are lumped at the center of mass and whose values are $m_{1}=4160 \mathrm{~kg}$ and $m_{2}=5910 \mathrm{~kg}$, respectively. Three degrees-offreedom (DOF) are assigned to each lumped mass: two horizontal translations in the direction of and perpendicular to the ground motion, and one rotation about a vertical axis passing through the center of mass. The translation in the direction of the ground motion, the translation perpendicular to the ground motion and the rotation are respectively denoted by $u_{1}, u_{2}, u_{3}$ for the first level, and $u_{4}, u_{5}, u_{6}$ for the second level. The response in each DOF was obtained from the measurements provided by displacement transducers and accelerometers.

\subsection{Overall response}

The overall response of the test structure was characterized by the formation of a strong column-weak beam mechanism. The structure remained almost elastic until the onset of seismic simulation $\mathrm{C} 200$, with the initial values of the fundamental period $T_{1}=0.31 \mathrm{~s}$ and damping ratio $\xi=2.7 \%$ remaining basically unchanged. At the end of seismic simulation $\mathrm{C} 200$ the period $T_{1}$ increased by about $70 \%$ and $\xi$ about $40 \%$, which reflects the occurrence of plastic deformations on the structure (damage). The enlargement of $T_{1}$ implies a drop in lateral stiffness of $35 \%$. About

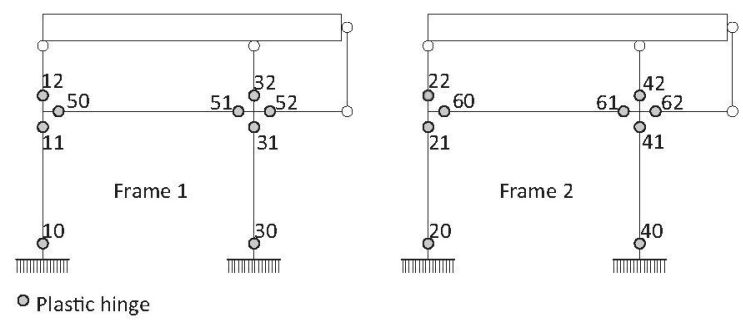

Fig. 7. Numbering of plastic hinges in the RC frames.

$8 \mathrm{~s}$ after the onset of seismic simulation $\mathrm{C} 300$, a video camera that focused on one of the beam ends recorded the formation of a flexural crack that opened abruptly at the beam end. This crack reached a maximum width of about $3 \mathrm{~mm}$; it was accompanied by a sudden vertical slide of approximately $10 \mathrm{~mm}$ between the two sides of the flexural crack. At this instant, severe concrete crashing was also observed at the column bases, and the lateral drift at the top of the specimen reached $4.06 \%$ of the total height. This is taken hereafter as the instant of collapse. Fig. 8 shows the complete history of lateral displacements in the direction of the base motion for the first, $u_{1}$, and second levels, $u_{4}$. Fig. 9 shows the corresponding history of rotations $u_{3}$ and $u_{6}$. It can be seen in the figures that the sign of the lateral displacement and of the rotation was the same in both levels. The motion plotted in Fig. 8 is dominated by the response in the first lateral translational mode, and that shown in Fig. 9 by the response in the first torsional mode.

\subsection{Characterization of the torsional response}

Table 1 shows the first translational, $T_{1}$, and the first torsional, $T_{\theta}$, periods, together with the damping ratio $\xi$, calculated after each seismic simulation. The table also shows the ratio $\Omega=T_{1} / T_{\theta}$ typically used to characterize the torsional behavior of the structure. The ratio $\Omega$ has an important influence on the torsional response. The smaller the ratio $\Omega$, the larger is the influence of the predominantly torsional mode of vibration on the response in the direction considered (compared to the predominantly translational mode) [18]. Some authors [18] denote structures with $\Omega>1$ as torsionally stiff and those with $\Omega<1$ as torsionally flexible. Other authors [2,3] define as torsionally flexible systems those with $\Omega<0.67$. Therefore the range of values for $\Omega$, which distinguish between the torsional flexible and torsional stiff structures is between 0.67 and 1 . According to the values obtained for $\Omega$, the test structure can be classified as a torsionally stiff system [2]. Values of $\Omega$ close to unity denote coupling between the lateral and torsional motions of the structure. The results of Table 1 indicate that, in contrast to $T_{1}$, which increases with the severity of the seismic simulation, $T_{\theta}$ remains unchanged during the tests. As a result, $\Omega$ tends to 


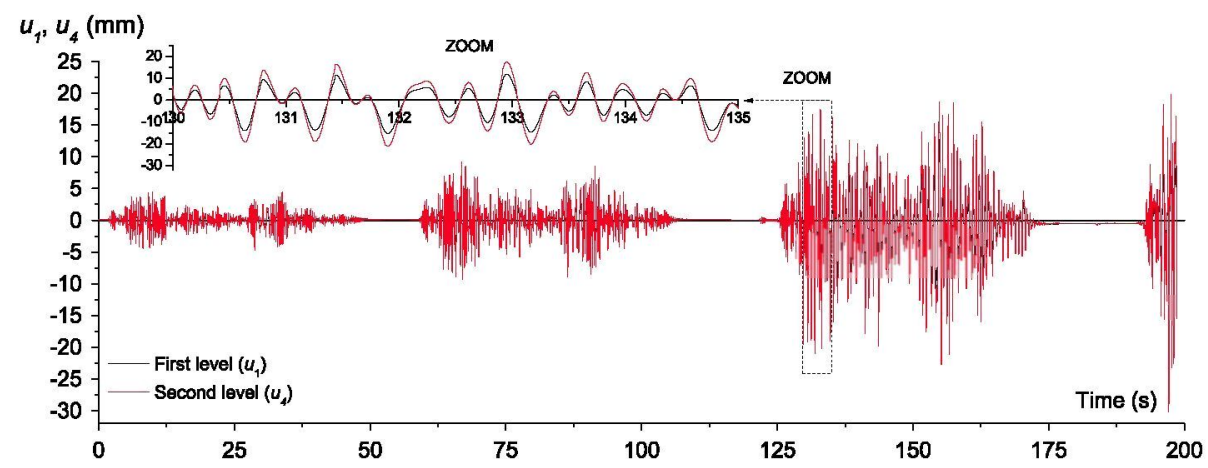

Fig. 8. History of lateral displacements in the direction of the base motion.

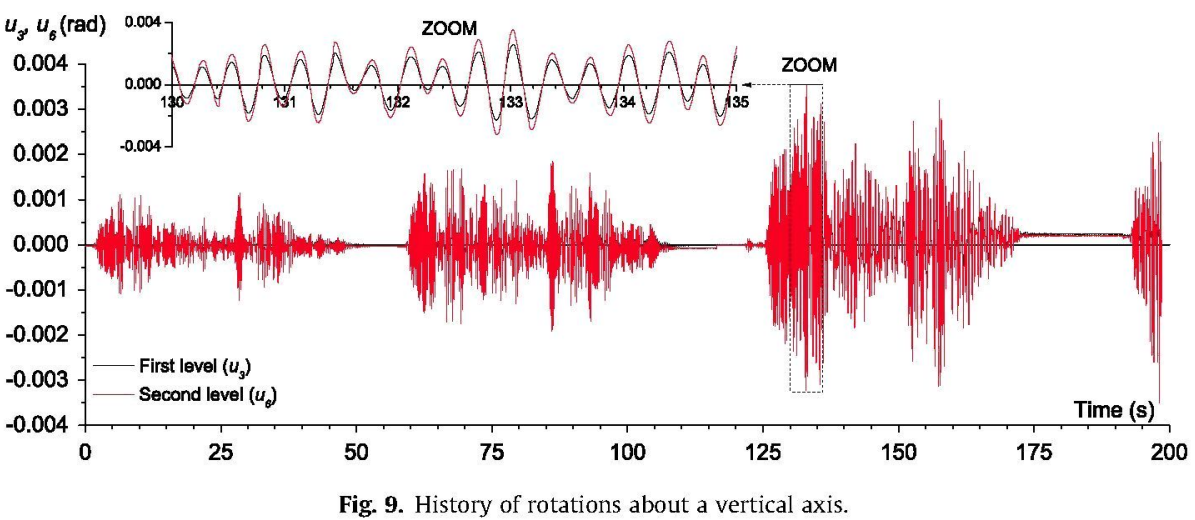

Table 1

Dynamic characterization.

\begin{tabular}{lllll}
\hline Seismic simulation & $T_{1}$ & $T_{\theta}$ & $\Omega=T_{1} / T_{\theta}$ & $\zeta$ \\
\hline C50 & 0.31 & 0.31 & 1.00 & 2.7 \\
C100 & 0.32 & 0.31 & 1.03 & 3.8 \\
C200 & 0.54 & 0.31 & 1.74 & 9.7 \\
C300 & - & - & - & -
\end{tabular}

increase and the coupling between lateral and torsional motions diminishes.

\subsection{Displacement of frames and rotation demands at floor level}

Table 2 summarizes the maximum response of the test structure in terms of displacements in the direction of the base motion and relative to the shaking table. The second to fifth columns show the peak lateral displacements, $d_{F 1, \max }$ and $d_{F 2, \max }$, at the first level of frames 1 and 2 (see Figs. 3 and 5 ) in the direction of shaking. The sixth and seventh columns show the peak rotation $u_{3, \max }$ of the first level of the test structure in the positive and negative domains; and the eighth column the absolute value of this rotation, $\left|u_{3}^{*}\right|$, at the instant when the lateral displacement $u_{1}$ of the first level in the direction of the base motion attained its peak value $\left|u_{1, \max }\right|$. Column nine shows the ratio $\Delta=\max \left\{\left|d_{F 1}\right|,\left|d_{F 2}\right|\right\} /\left|u_{1, \max }\right|$, where $\left|d_{F 1}\right|$ and $\left|d_{F 2}\right|$ are the lateral displacements in the direction of the ground motion at the first level of frames 1 and 2, respectively, at the instant when $\left|u_{1, \max }\right|$ was reached. The parameter $\Delta$ characterizes the increase in frame displacement due to torsion. It is worth noting that $\Delta$ has been defined according to the displacement of the center of mass for the purpose of comparison with other studies.

It is noted in Table 2 that peak displacements of frame 2 are significantly larger than those of frame 1 , especially during the seismic simulations $\mathrm{C} 50$ and $\mathrm{C} 100$, indicating that frame 1 is stiffer than frame 2. It is worth noting that during seismic simulations $\mathrm{C} 50$ and $\mathrm{C} 100$ the strains in the reinforcing bars remained below or slightly above (less than two times) the yield strain, as discussed later in Section 3.5. However, concrete cracking was clearly observed, which necessarily degraded the lateral stiffness of the frames. An uneven degradation of this lateral stiffness between the two frames and uncertainties in the building process could explain the different displacements of the frames due to torsion effects. The second source of torsional effects is judged to be of relatively minor importance, since the test model was built in the laboratory, and both the construction process and the set-up were carefully controlled.

Comparing $\left|u_{3}^{*}\right|$ with $u_{3, \max }{ }^{+}$and $u_{3, \max }{ }^{-}$, it is clear that the maximum rotation did not occur at the instant when the first level of the structure attained its peak lateral displacement. The rotation of the first floor level increased with shaking intensity. Unlike floor rotation, the increase in the frame displacement demand due to torsion, $\Delta$, decreases as the shaking is more intense and the structure enters the nonlinear range. The effects of torsion on the displacement demand diminish when the response is nonlinear (13\% and $4 \%$ for rare and very rare earthquakes) in comparison with the values exhibited when the specimen remains elastic (up to $33 \%$ ). Similar values of $\Delta$ (around 1.3 ) are reported by Marušić and Fajfar [6] for RC frame structures. This behavior can be attributed to the fact that, with yielding, the structure becomes torsionally stiffer in comparison to the lateral stiffness. This is supported by the increase in the ratio of translational to torsional periods $\Omega$ shown in Table 1.

\subsection{Strain demand in the reinforcing bars}

Table 3 shows the normalized peak strains, $\varepsilon / \varepsilon_{y}$, measured in the reinforcing bars at the sections where the plastic hinges developed. 
Table 2

Peak response at the first floor level.

\begin{tabular}{|c|c|c|c|c|c|c|c|c|}
\hline \multirow[t]{2}{*}{ Seismic simulation } & \multicolumn{2}{|l|}{ Frame 1} & \multicolumn{2}{|l|}{ Frame 2} & \multirow[b]{2}{*}{$u_{3, \max }{ }^{+}\left(\times 10^{-3} \mathrm{rad}\right)$} & \multirow[b]{2}{*}{$u_{3, \max }{ }^{-}\left(\times 10^{-3} \mathrm{rad}\right)$} & \multirow[b]{2}{*}{$\left|u_{3}^{*}\right|\left(\times 10^{-3} \mathrm{rad}\right)$} & \multirow[b]{2}{*}{$\Delta$} \\
\hline & $d_{F 1, \max }^{+}(\mathrm{mm})$ & $d_{F 1, \max }{ }^{-}(\mathrm{mm})$ & $d_{F 2, \max }{ }^{+}(\mathrm{mm})$ & $d_{F 2, \max }-(\mathrm{mm})$ & & & & \\
\hline $\mathrm{C} 50$ & 2.23 & -1.94 & 3.44 & -3.14 & 0.88 & -0.93 & 0.80 & 1.33 \\
\hline$C 100$ & 4.69 & -4.02 & 6.43 & -6.28 & 1.40 & -1.46 & 1.37 & 1.27 \\
\hline$C 200$ & 11.15 & -10.45 & 14.56 & -10.78 & 2.59 & -2.31 & 1.70 & 1.13 \\
\hline$C 300$ & 80.12 & -61.53 & 82.82 & -61.87 & 2.66 & -3.70 & 2.12 & 1.04 \\
\hline
\end{tabular}

Table 3

Strain demand in the reinforcing steel.

\begin{tabular}{|c|c|c|c|c|c|c|c|c|c|c|c|c|c|c|c|}
\hline \multicolumn{4}{|l|}{$\mathrm{C50}$} & \multicolumn{4}{|l|}{$\mathrm{C} 100$} & \multicolumn{4}{|l|}{$\mathrm{C} 200$} & \multicolumn{4}{|l|}{$\mathrm{C} 300^{\mathrm{a}}$} \\
\hline \multicolumn{2}{|c|}{ Frame1 } & \multicolumn{2}{|c|}{ Frame 2} & \multicolumn{2}{|c|}{ Frame 1} & \multicolumn{2}{|c|}{ Frame 2} & \multicolumn{2}{|c|}{ Frame 1} & \multicolumn{2}{|c|}{ Frame 2} & \multicolumn{2}{|c|}{ Frame 1} & \multicolumn{2}{|c|}{ Frame 2} \\
\hline Hinge & $\varepsilon / \varepsilon_{y}$ & Hinge & $\varepsilon / \varepsilon_{y}$ & Hinge & $\varepsilon / \varepsilon_{y}$ & Hinge & $\varepsilon / \varepsilon_{y}$ & Hinge & $\varepsilon / \varepsilon_{y}$ & Hinge & $\varepsilon / \varepsilon_{y}$ & Hinge & $\varepsilon / \varepsilon_{y}$ & Hinge & $\varepsilon / \varepsilon_{y}$ \\
\hline 10 & 0.48 & 20 & 0.63 & 10 & 0.98 & 20 & 1.92 & 10 & 6.37 & 20 & 4.90 & 10 & $>15$ & 20 & 5.20 \\
\hline 30 & 0.55 & 40 & 0.69 & 30 & 1.12 & 40 & 1.44 & 30 & 6.98 & 40 & 6.11 & 30 & 7.20 & 40 & 6.20 \\
\hline 50 & 0.34 & 60 & 0.52 & 50 & 0.79 & 60 & 0.99 & 50 & 2.83 & 60 & 6.23 & 50 & $>15$ & 60 & $>15$ \\
\hline 51 & 0.29 & 61 & 0.39 & 51 & 0.70 & 61 & 0.79 & 51 & 3.28 & 61 & 2.08 & 51 & 6.20 & 61 & 7.20 \\
\hline 52 & 0.26 & 62 & 0.40 & 52 & 0.56 & 62 & 0.67 & 52 & 1.65 & 62 & 2.42 & 52 & 2.50 & 62 & 6.50 \\
\hline
\end{tabular}

a Until the instant of collapse.

This ratio $\varepsilon / \varepsilon_{y}$ characterizes the ductility demand in the reinforcing bars. Each row in Table 3 contains the information on two hinges located at the same relative position in frames 1 and 2 (i.e. hinges 10 and 20; hinges 30 and 40, and so on, in Fig. 7). As a result of the different lateral displacements of frames 1 and 2 discussed in Section 3.4 , systematically larger peak strains were measured in the rebars of frame 2 during seismic simulations $\mathrm{C} 50$ and $\mathrm{C} 100$. It must be underlined that in simulation $\mathrm{C} 100$ some rebars at the column bases of frame 2 (hinges 20-40) reached strains that are twice the yield strain and up to two times the values found in symmetric positions in the stiffer frame 1.

The seismic response of the test structure during seismic simulations C200 and C300 was highly inelastic. All the hinges involved in the weak-beam strong-column plastic mechanism (hinges 10 , $20,30,40,51,52,60,61$ and 62) showed strains that exceeded the yield strain from about 2 to 7 times in case of simulation C200, and from 2.5 to more than 15 times in simulation $\mathrm{C} 300$. However, in contrast to seismic simulations $\mathrm{C} 50$ and $\mathrm{C} 100$, in seismic simulations $\mathrm{C} 200$ and $\mathrm{C} 300$ the trend of systematically larger peak strains in frame 2 than in frame 1 was not observed. Indeed, the ratios $\varepsilon / \varepsilon_{y}$ in the hinges located at the same positions in frames 1 and 2 are similar in several cases. This observation is consistent with the fact pointed out in Section 3.4 that the ratio $\Delta$ is close to 1 , and supports the assertion that torsion effects are of minor importance when the structure largely enters the nonlinear range.

\subsection{Cumulative plastic deformation demand in the plastic hinges}

The amount of hysteretic energy (cumulative damage) dissipated by each plastic hinge $W_{p k}$ at any instant of the loading process was estimated from measurements provided by strain gages on the basis of the simplified method in reference [32] where total dissipated energy in RC components is the sum of the energies dissipated by concrete and steel rebars. First, the depth of the sections was discretized in fibers. The history of strain at each fiber was approximated by assuming that plane sections remain plane. Second, the history of stress at each fiber can be evaluated from the materials constitutive laws for confined concrete and steel reinforcement. Hysteretic energy dissipated by each fiber can be calculated as the integral of the stress-strain curves along the plastic hinge length. Finally, total hysteretic energy dissipated by a plastic hinge $W_{p k}$ can be obtained as the sum of the latter.
For each plastic hinge, Table 4 shows the values of $W_{p k}$ accumulated through the successive seismic simulations. The last two rows in Table 4 show the hysteretic energy dissipated by each frame, expressed in $\mathrm{Nm}$ and as percentage of the total energy dissipated by the two frames. It is observed that for low levels of seismic hazard (simulations $\mathrm{C} 50$ and $\mathrm{C} 100$ ), although the cumulative damage is limited (as expected), it is from 3 to 5 times larger in frame 2 than in frame 1 . For the seismic hazard associated with the design earthquake (simulation $\mathrm{C200}$ ) for which the structure is allowed to undergo significant damage, the cumulative damage in frame 2 is about three times larger in than in frame 1; but the difference tends to vanish as the severity of the ground motion increases, becoming negligible for the maximum credible earthquake (simulation C300) for which the structure is on the brim of collapse.

These results are consistent with the observations of Sections 3.4 and 3.5, and suggest that torsion effects become negligible when assessing the performance of the structure near collapse.

\subsection{Damage at the plastic hinge level and in each frame}

The damage on the plastic hinges of the RC frames is related to the cumulative plastic deformation (hysteretic energy) addressed in Section 3.6 and to the maximum rotation demand relative to the yield rotation. The well-known index of damage proposed by Park and Ang [33], $D I_{P A, k}$, takes into account the contribution of both aspects, and it is defined as follows:

$D_{P A, k}=0.5\left[\frac{\theta_{m}^{+}-\theta_{y}^{+}}{\theta_{u}^{+}-\theta_{y}^{+}}+\frac{\left|\theta_{m}^{-}\right|-\left|\theta_{y}^{-}\right|}{\left|\theta_{u}^{-}\right|-\left|\theta_{y}^{-}\right|}\right]+\beta \frac{W_{p k}}{0.5\left(M_{y}^{+} \theta_{u}^{+}+\left|M_{y}^{-} \| \theta_{u}^{-}\right|\right)}$

Here, $\theta_{y}$ is the chord rotation at yielding and $\theta_{u}$ the ultimate rotation, which can be predicted with the equations recommended by Eurocode 8-Part 3 (Annex A) [30], based on the work by Fardis [34]. $M_{y}$ is the bending moment at yielding, estimated by analytical formulae.

Table 5 shows the values of $D I_{P A, k}$ calculated in each plastic hinge. It can be seen that $D I_{P A, k}$ is basically null for seismic simulations $C 50$ and $C 100$. For the higher levels of seismic hazard $C 200$ and $\mathrm{C} 300$, there are no relevant differences in the damage endured by hinges located in the same positions in frames 1 and 2 . The damage at the frame level was also calculated using an approach 
Table 4

Hysteretic energy dissipated by the plastic hinges (units: Nm).

\begin{tabular}{|c|c|c|c|c|c|c|c|c|c|c|c|c|c|c|c|}
\hline \multicolumn{4}{|l|}{ C50 } & \multicolumn{4}{|l|}{$\mathrm{C} 100$} & \multicolumn{4}{|l|}{$\mathrm{C} 200$} & \multicolumn{4}{|l|}{$\mathrm{C} 300^{\mathrm{a}}$} \\
\hline \multicolumn{2}{|c|}{ Frame 1} & \multicolumn{2}{|c|}{ Frame 2} & \multicolumn{2}{|c|}{ Frame 1} & \multicolumn{2}{|c|}{ Frame 2} & \multicolumn{2}{|c|}{ Frame 1} & \multicolumn{2}{|c|}{ Frame 2} & \multicolumn{2}{|c|}{ Frame 1} & \multicolumn{2}{|c|}{ Frame 2} \\
\hline Hinge & $W_{p k}$ & Hinge & $W_{p k}$ & Hinge & $W_{p k}$ & Hinge & $W_{p k}$ & Hinge & $W_{p k}$ & Hinge & $W_{p k}$ & Hinge & $W_{p k}$ & Hinge & $W_{p k}$ \\
\hline 10 & 2.3 & 20 & 9.8 & 10 & 3.8 & 20 & 27.6 & 10 & 262.8 & 20 & 258.9 & 10 & 316.6 & 20 & 464.3 \\
\hline 30 & 1.7 & 40 & 8.9 & 30 & 3.4 & 40 & 16.9 & 30 & 69.4 & 40 & 402.0 & 30 & 109.5 & 40 & 728.4 \\
\hline 50 & 1.4 & 60 & 1.2 & 50 & 2.5 & 60 & 2.5 & 50 & 17.8 & 60 & 256.0 & 50 & 957.3 & 60 & 256.0 \\
\hline 51 & 2.8 & 61 & 1.6 & 51 & 2.8 & 61 & 2.4 & 51 & 17.3 & 61 & 6.7 & 51 & 106.6 & 61 & 121.3 \\
\hline 52 & 1.9 & 62 & 7.5 & 52 & 2.6 & 62 & 18.3 & 52 & 5.3 & 62 & 173.3 & 52 & 71.1 & 62 & 237.6 \\
\hline \multirow[t]{2}{*}{ Total } & 10.1 & & 29.0 & & 15.1 & & 67.7 & & 372.6 & & 1096 & & 1561 & & 1808 \\
\hline & $26 \%$ & & $74 \%$ & & $18 \%$ & & $82 \%$ & & $25 \%$ & & $75 \%$ & & $46 \%$ & & $54 \%$ \\
\hline
\end{tabular}

Table 5

Damage at the local (plastic hinge) and frame levels.

\begin{tabular}{|c|c|c|c|c|c|c|c|c|c|c|c|c|c|c|c|}
\hline \multicolumn{4}{|l|}{$\mathrm{C} 50$} & \multicolumn{4}{|l|}{$\mathrm{C} 100$} & \multicolumn{4}{|l|}{$\mathrm{C} 200$} & \multicolumn{4}{|l|}{$\mathrm{C} 300^{\mathrm{a}}$} \\
\hline \multicolumn{2}{|c|}{ Frame 1} & \multicolumn{2}{|c|}{ Frame 2} & \multicolumn{2}{|c|}{ Frame 1} & \multicolumn{2}{|c|}{ Frame 2} & \multicolumn{2}{|c|}{ Frame 1} & \multicolumn{2}{|c|}{ Frame 2} & \multicolumn{2}{|c|}{ Frame 1} & \multicolumn{2}{|c|}{ Frame 2} \\
\hline Hinge & $D I_{P A}$ & Hinge & $D I_{P A}$ & Hinge & $D I_{P A}$ & Hinge & $D I_{P A}$ & Hinge & $D I_{P A}$ & Hinge & $D I_{P A}$ & Hinge & $D I_{P A}$ & Hinge & $D I_{P A}$ \\
\hline 10 & 0.00 & 20 & 0.00 & 10 & 0.00 & 20 & 0.01 & 10 & 0.22 & 20 & 0.21 & 10 & 1.09 & 20 & 1.14 \\
\hline 30 & 0.00 & 40 & 0.00 & 30 & 0.00 & 40 & 0.01 & 30 & 0.16 & 40 & 0.26 & 30 & 1.03 & 40 & 1.22 \\
\hline 50 & 0.00 & 60 & 0.00 & 50 & 0.00 & 60 & 0.00 & 50 & 0.14 & 60 & 0.22 & 50 & 1.28 & 60 & 1.05 \\
\hline 51 & 0.00 & 61 & 0.00 & 51 & 0.00 & 61 & 0.00 & 51 & 0.14 & 61 & 0.14 & 51 & 1.00 & 61 & 1.01 \\
\hline 52 & 0.00 & 62 & 0.00 & 52 & 0.00 & 62 & 0.01 & 52 & 0.14 & 62 & 0.19 & 52 & 1.00 & 62 & 1.05 \\
\hline Frame & 0.00 & & 0.00 & & 0.00 & & 0.01 & & 0.18 & & 0.23 & & 1.19 & & 1.14 \\
\hline
\end{tabular}

similar to that applied by Park et al. [33]. These authors estimated the overall building damage as the sum of the damage index of each component $i$ weighted by an energy absorbing contribution factor, which represents the ratio between the energy absorbed by the component, $E_{i}$, and the total energy absorbed by all components, $\Sigma E_{i}$. Similarly, in this case, the overall damage index of each frame $D I_{P A, F}$, is given by:

$D I_{P A, F}=\sum_{k=1}^{p} D I_{P A, k} \frac{W_{p k}\left(t_{o}\right)}{\sum_{i=1}^{p} W_{p i}\left(t_{0}\right)}$

This sum extends to the total number $p$ of plastic hinges in the frame. The results are shown in the last row of Table 5. It is seen that the damage on the frames is negligible for seismic simulations C50 and $\mathrm{C} 100$, and very similar in frames 1 and 2 for the seismic simulations $\mathrm{C} 200$ and $\mathrm{C} 300$

\section{Conclusions}

The torsional seismic response of a nominally symmetric reinforced concrete frame structure in the direction of the seismic loading is discussed by analyzing the histories of displacement and strains recorded during a sequence of uniaxial shaking table tests. The source of the torsional response is attributed to uncertainties in the building process and to non-uniform yielding of structural components. The latter source is believed to be more relevant, since the building process of the test structure and the set-up of the experiments were carefully controlled. It is found that:

1. The lengthening of periods due to yielding as the level of damage on the structure increases, is different for the translational and for the torsional motions. More precisely, the ratio $\Omega$ between translational and rotational periods increases up to about two times, from the initial quasi-elastic state to the near-collapse state. This causes the structure to become torsionally stiffer (in comparison to the lateral stiffness) as the level of plastic deformation increases. As a result, the importance of torsion effects diminishes as the level of damage on the structure increases.

2. For low levels of seismic hazard, for which the structure is designed to perform basically in the elastic range (with minor damage), accidental eccentricity increases the lateral displacement demand in the outermost frames of the structure up to about $30 \%$, increases the strains in the reinforcing bars up to two times, and also increases the energy dissipated by the plastic hinges (mainly due to plastic deformation of concrete) up to five times. Since the strains in the reinforcing bars remain in most cases below the yield strain and the energy dissipated by the hinges is attributed to concrete, for low levels of seismic hazard the torsion effects are not a concern for the health of the structure. However, the significant increase of displacement demand on the frames might cause significant damage to non-structural components.

3. For high levels of seismic hazard, for which the structure is designed to undergo significant plastic deformations, the effect of accidental torsion becomes negligible from the point of view of the level of damage endured by the plastic hinges, or the ductility demands on the reinforcing rebars.

4. Pending the results of additional studies, the findings reported suggest that accidental eccentricity needs to be considered in the evaluation of the performance levels corresponding to very frequent or frequent earthquakes (for which the damage to non-structural components is a matter of concern). As noted above, in this study the source of the torsional response is attributed mainly to non-uniform yielding of structural components. Since the source of the accidental eccentricity is also the mass distribution, amplifications in case of very frequent earthquakes may be much larger than the $33 \%$ found in this study. However, accidental eccentricity could be disregarded when evaluating the performance levels corresponding to rare (design) or very rare earthquakes. 
5. Finally, as a fundamental premise of this experimental investigation, it is worth noting that the purpose of the tests was to study the behavior of the partial structural model under earthquake-type dynamic loading. It was not attempted to reproduce the particular response that the partial structural model would experience inside the overall frame, which is influenced by dynamic interactions with the upper part of the structure.

\section{Acknowledgements}

This work received financial support from the Spanish Government under project BIA2011-26816 and from the European Union (Feder). 